\title{
Charcot Leyden Crystals in Liver FNAC
}

\author{
Lakshmi Agarwal ${ }^{1 *}$, Ila Sharma² and Naresh Rai $^{2}$ \\ ${ }^{1}$ Dept. of Pathology, Pushpadi Cancer care Centre \& Govt. Medical College, Kota, India \\ ${ }^{2}$ Dept. of Pathology, Govt. Medical College, Kota, India
}

\section{Dear sir,}

Charcot Leyden Crystals (CLCs) are hexagonal bipyramidal crystalline structures which are pathognomic feature of eosinophilic leucocyte infiltration. The CLCsprotein which forms these crystals is a major auto crystallizing constituent of eosinophils and basophils ${ }^{1}$. It has Lysophospholipase activity. These crystals can be seen in trichrome-stained preparations of faeces examined for enteric parasites, in respiratory secretions or more rarely in tissue biopsies. Conditions associated with CharcotLeyden crystal formation include- gastrointestinal parasite infections- amoebic/parasitic; allergic diseases- eg. Asthma; haematological and neoplastic conditions (rarely) - Eosinophilicleukemia, Prostatic carcinoma ${ }^{2}$

A 42 year female patient presented with a hepatic mass. She also complained of fever, pain and weight loss. Her routine hematological analysis was as follows: hemoglobin - $9.6 \mathrm{~g} / \mathrm{dL}$, hematocrit - $28.8 \%$, total leucocyte count 13,700/mm3, differential leukocyte count - polymorphs: $56 \%$, lymphocyte: $19 \%$, eosinophil: $20 \%$, monocyte: $5 \%$. Liver function test was found to be within normal limits. On USG, it was considered a neoplastic lesion. CT scan was not done.

FNAC was done from the liver mass. The smears showed necrosis with many eosinophils, few neutrophils, macrophages \& occasional mast cells. Scanty small clusters of hepatocytes were seen. Many Charcot Leyden Crystals were seen all along the smears (Figure 1,2,). No trophozoites of E. histolytica were seen. The stain for Acid fast bacilli was found to be negative.

The presence of Charcot Leyden Crystals showed eosinophilic abscess, thus patient was advised ELISA/ IHA test for amoeba and stool examination for ova/cysts. The patient was treated with antihelminthic drugs and she responded well.

The finding of Charcot Leyden Crystals on FNAC of Liver is a rare occurrence.It was first described by Charcot and Robin ${ }^{2},{ }^{3}$ in 1853 as peculiar crystals which they had found in the cardiac blood and the spleen of a leukemic patient. Leyden $^{3}$ in 1872 recognized the same crystals in asthmatic sputum. They appear as slender needles of uniform shape and since then it is known as Charcot Leyden crystals.
Askanazy ${ }^{4}$ observed the relation between CLCs and eosinophilia and concluded that intimate chemical relations exist between eosinophilicmyelocytes and leucocytes and Charcot leyden crystals.

The most common clinical presentation is fever, pain, pruritus, rash or urticaria and weight loss. Hepatosplenomegaly can also be found. But the diagnostic finding is increased eosinophil count on peripheral blood for a long time.

Hepatic mass in an adult can pose a diagnostic dilemma. The various differential diagnoses which can be considered are adenoma, primary and secondary malignancy of liver, inflammatory pseudotumor, and eosinophilic granuloma. The neoplastic lesion of liver was excluded due to the absence of dysplastic hepatocytes and malignant cells. A possibility of 'eosinophilic granuloma' (Langerhans cell histiocytosis) which may also show numerous Charcot Leyden crystals in a background of eosinophils, ${ }^{5}$ was ruled out due to the absence of charactersticgrooved nuclei cells. The inflammatory pseudotumor ${ }^{6}$ is a benign lesion and reveals intense inflammation comprising neutrophils, eosinophils, macrophages and lymphocytes along with CLCs. The etiology is unknown but infection can be the possible cause.

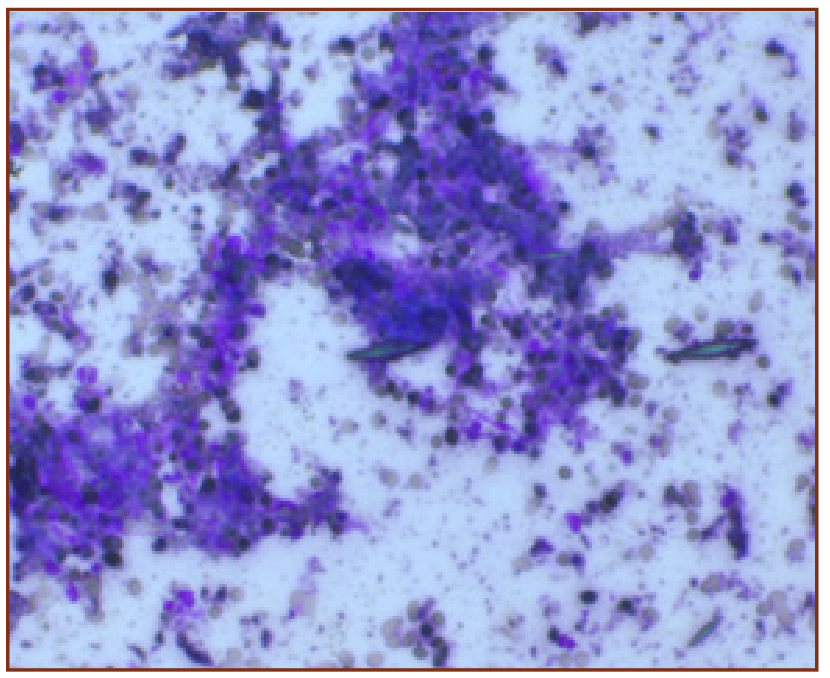

Fig.1: Giemsa stain, 10X showing Exudate, Necrosis, CLCs, Inflammatory infiltrate. 


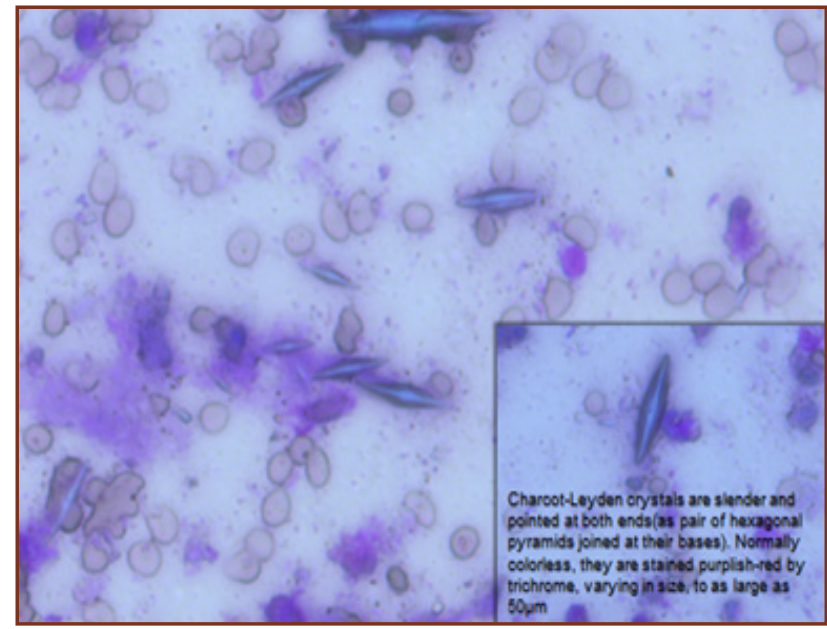

Fig. 2: Giemsa stain, 40X showing abundant CLCs.

The CLCs when found is a proof of eosinophilic inflammation and can be used to rule out pyogenic abscess and diagnose a parasitic infection of the liver. Parasitic infestation of the liver is an extremely rare presentation and may pose a significant challenge in its diagnosis and management. Specific tests to diagnose the parasite can thus be undertaken.
FNAC is very cost effective and can play an important role in diagnosing such cases. In the present case, the FNAC clearly ruled out a neoplastic condition or bacterial infection, leading to proper diagnosis and treatment of the patient.

\section{References}

1. Misra V, Debnath S, Misra P, Singh PA. Significance of Charcot Leyden crystals in hepatic aspirates. J Cytol. 2009 Apr-Jun; 26(2): 77-79

2. Charcot J, Robin C. Observation de leucocythemie. CR MemSocBiol 1853;5:454-450

3. Dor PJ, Ackerman SJ, Gleich GJ (1984) Charcot-Leyden crystal protein and eosinophil granule major basic protein in sputum of patients with respiratory diseases. Am Rev Respir Dis 130: 1072-1077.

4. Askanazy, M. in Henke F. and Lubarsch, 0.Handbuch der spez. path. Anatomie und Histologie, Berlin, 1937, Knochenmark. Vol. I part 2: p. 789, Julius Springer.

5. Arora VK, Singh N, Bhatia A. Charcot Leyden crystals in fine needle aspiration cytology. ActaCytol. 1997;41:409-12

6. Ryan RS, Al-Hashimi H, Lee MJ. Hepatic abscesses in elderly patients mimicking metastatic disease. Ir J Med Sci 2001; 170:251-3.

*Corresponding author:

Dr Lakshmi Agarwal, Consultant Onco-Pathologist, AP, Dept. of Pathology, Pushpadi Cancer care Centre \& Govt. Medical College, Kota, India

Phone: +91 09772464587

Email: drlaxmiagarwal@gmail.com

Financial or other Competing Interests: None. 